American Journal of Applied Sciences 3 (12): 2147-2150, 2006

ISSN 1546-9239

(c) 2006 Science Publications

\title{
Remote Personal Tracking System (RPTS)
}

\author{
Abdfatah A. Yahya and Mahmoud Z. Iskandarani \\ Faculty of Science and Information Technology, Al-Zaytoonah University of Jordan \\ P O BOX: 911597, Post Code: 11191, Amman-Jordan
}

\begin{abstract}
A novel system for remote tracking of personnel was proposed. The system was based on assuring that the moving tracked code belongs to the actual person assigned such a code by smelling and temperature measuring the person who holds such a device. The system would operate using a specific RF signal and allows for an accurate, economic and secure environment in which diverse of people can interact together.
\end{abstract}

Key words: Positioning, sensor networks, coverage, localization, wireless networks, mobile systems

\section{INTRODUCTION}

The traditional computing environment that requires users to come to a wired computer may be ineffective or inefficient in many situations. The solution is to make computers small enough that they are easy to carry or even to wear. Such mobile devices can communicate with traditional systems and infrastructure via wireline or, even better, wireless networks. The ability to communicate and collaborate any time and from anywhere provides organizations with strategic advantage by increasing productivity, speed and customer service.

Mobile and wireless computing provide the infrastructure for mobile, any time and from any place. They enable location-based knowledge, which is based on knowing where people are at any given time and on the ability to communicate with them. Mobile and wireless computing are changing how IT is deployed and are creating the foundations of the futuristic computing environment called pervasive computing ${ }^{[1-5]}$.

Wireless Sensor Networks have been proposed for a multitude of location-dependent applications. For such systems, the cost and limitations of the hardware on sensing nodes prevent the use of range-based localization schemes that depend on absolute point-topoint distance estimates. Because coarse accuracy is sufficient for most sensor network applications, solutions in Adaptable Locators (AL) are being pursued as a cost-effective alternative to more expensive current approaches such as GPS.

Wireless Controlled Networks differs from other traditional wireless or wired networks through sensor and actuator based system that operates within a specified environment. Such networks have been proposed for various applications including search and rescue, disaster relief, target tracking and smart environments. The inherent characteristics of these sensor networks make a node's location an important part of their state. For such networks, location is being used to identify the location at which sensor readings originate, (for example, identifying a target's position during tracking using novel communication protocols that route to geographical areas instead of ID ${ }^{[6-10]}$.

In this paper, a new approach for personal tracking and security control is presented. This proposed system makes use of state of the art technologies that for the first time include biological fingerprinting as part of its tracking system.

Design considerations: Providing a reliable technology and architecture for determining the location of real world objects and people will undoubtedly enable applications, customization and inference not currently possible. There have been many systems and architectures over the years tackling the problem of determining location. Since each was developed to fulfill a different goal, they vary widely in many parameters including accuracy, cost, size, configurability, security and reliability. Examples include GPS, Active Badges and the Xerox ParcTAB, AT\&T Cambridge Ultrasonic Bats, Microsoft Research's WaveLAN system, the Smart Floor from Georgia Tech, PinpointCo's radio tags, various computer vision systems and various cellular phone based solutions.

Hence, Sensor Networks are considered now to be an important area within the technology of networking. It could include hundreds of inexpensive nodes, each having communication and computational power. Storage and processing power need not to be a limiting factor if such nodes are used only to report activities in a dynamic manner (on line) rather than to store and report.

One of the challenges faced is energy consumption by such nodes and the need to recharge them at certain periods of time. This can be addressed through design of both hardware and software.

Corresponding Author: Abdfatah A. Yahya, Faculty of Science and Information Technology, Al-Zaytoonah University of Jordan, P O BOX: 911597, Post Code: 11191, Amman-Jordan 
The location sensing architecture envisaged is conceptually simple even though the algorithm implementation is somewhat solid. Two base stations provide signal strength measurements mapping to an approximate distance. A central server then calculates the values to establish position of the tagged object using the following equations.

$\mathrm{d}_{1}^{2}=\mathrm{X}_{\mathrm{i}}^{2}+\mathrm{Y}_{\mathrm{i}}^{2}$

$\mathrm{d}_{2}{ }^{2}=\left(\mathrm{a}-\mathrm{X}_{\mathrm{i}}\right)^{2}+\left(\mathrm{b}-\mathrm{Y}_{\mathrm{i}}\right)^{2}$

From 1 and 2

$\mathrm{m}=\frac{\mathrm{d}_{2}^{2}-\mathrm{d}_{1}^{2}-\mathrm{a}^{2}-\mathrm{b}^{2}}{2 \mathrm{a}}$

We can now find the values of $\mathrm{X}$ and $\mathrm{Y}$ :

$\mathrm{X}=\mathrm{m}-\frac{\mathrm{b}}{\mathrm{a}} \mathrm{Y}$

$\mathrm{Y}=\frac{\left(\left(2 \mathrm{~b} / \mathrm{a}+-\left(4 \mathrm{~b}^{2} \mathrm{~m}^{2} / \mathrm{a}^{2}\right)-8\left(\mathrm{~m}^{2}+\mathrm{d}_{1}^{2}\right)\right)\right.}{4}$

Where: $(\mathrm{X}, \mathrm{Y})$ and $(\mathrm{a}, \mathrm{b})$ : Tracked Point

Coordinate (TPC)

And: $\mathrm{d} 1, \mathrm{~d} 2$ are the measured distance using signal strengths

Finally, the computed object positions are sent to a central server for further application processes. The general layout of the proposed system is shown in Fig. 1.

The operational algorithm: Many locator algorithms for sensor based networks have been proposed to provide per-node location information. With regard to the mechanisms used for estimating location, such algorithms divide protocols into two categories: rangebased and range-free. The former is defined by protocols that use absolute point-to-point distance estimates (range) or angle estimates for calculating location. The latter makes no assumption about the availability or validity of such information. Our rangebased algorithm is divided into two parts; the transmitter and the receiver ${ }^{[11-15]}$. The operating algorithms are illustrated in Fig. 2 and 3.

The illustrated algorithm provides a comprehensive way to track and protect people using multidimensional data collection technique. The technique is based on using a triangular sensing Element system or what we call (TSE). Such a sensor-based detection system consists of the following:

1. Odor detector chip: Instruments which electronically simulate the human olfactory response using arrays of dissimilar sensors have become known as eNoses ${ }^{[16,17]}$. The general idea is patterned upon a human who has 10 million smell receptors in their noses.

Faster qualitative and quantitative analyses of chemical compounds are becoming a necessity in

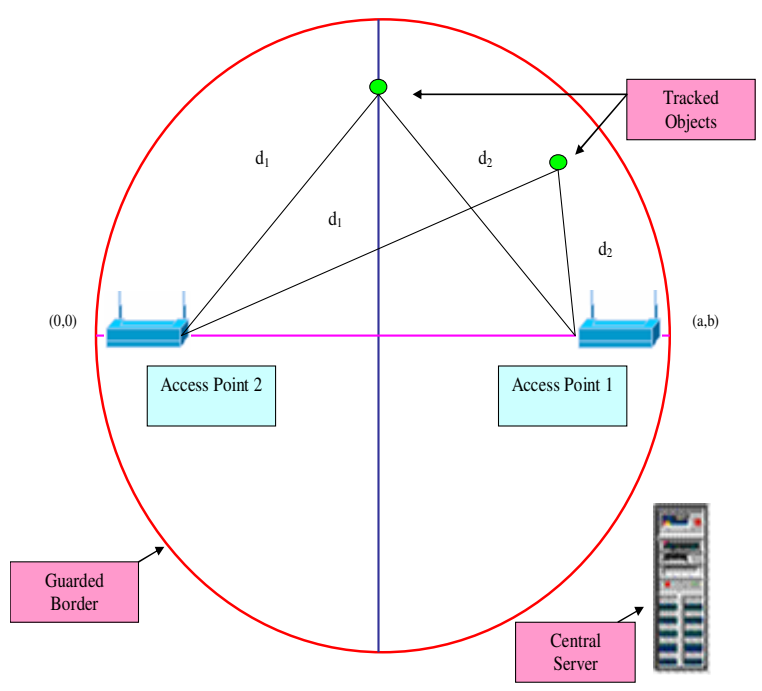

Fig. 1: Tracking system layout

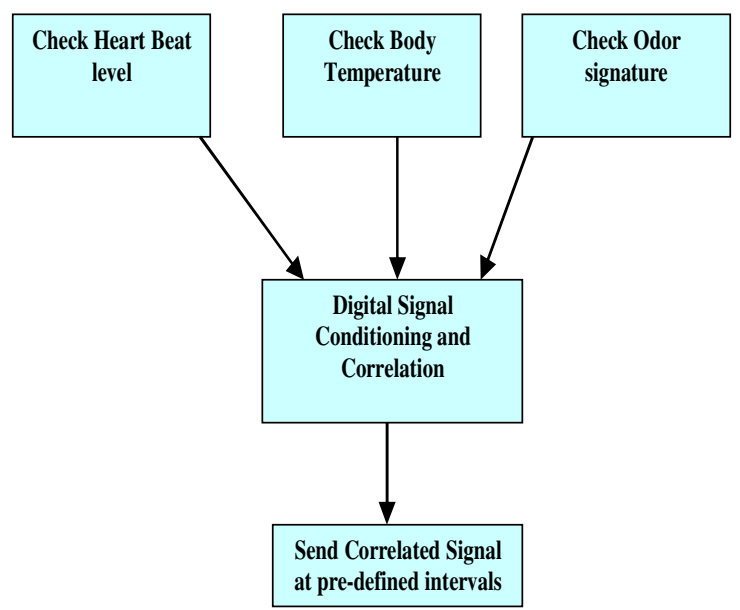

Fig. 2: Algorithm for transmitting device

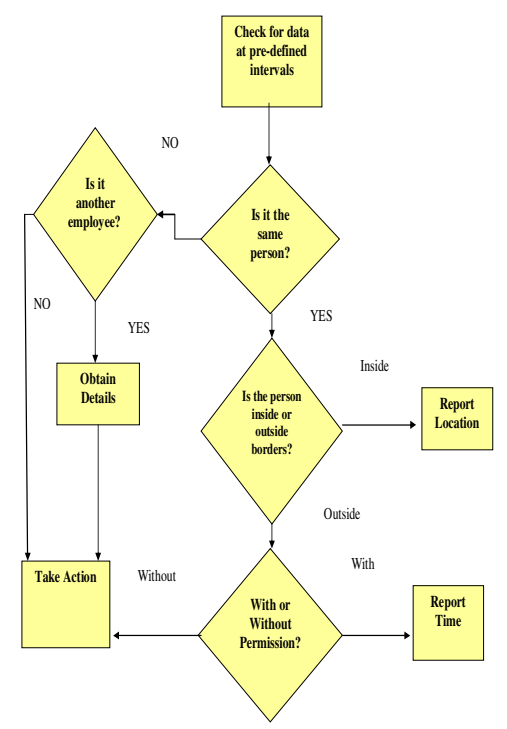

Fig. 3: Algorithm for receiving and processing system 


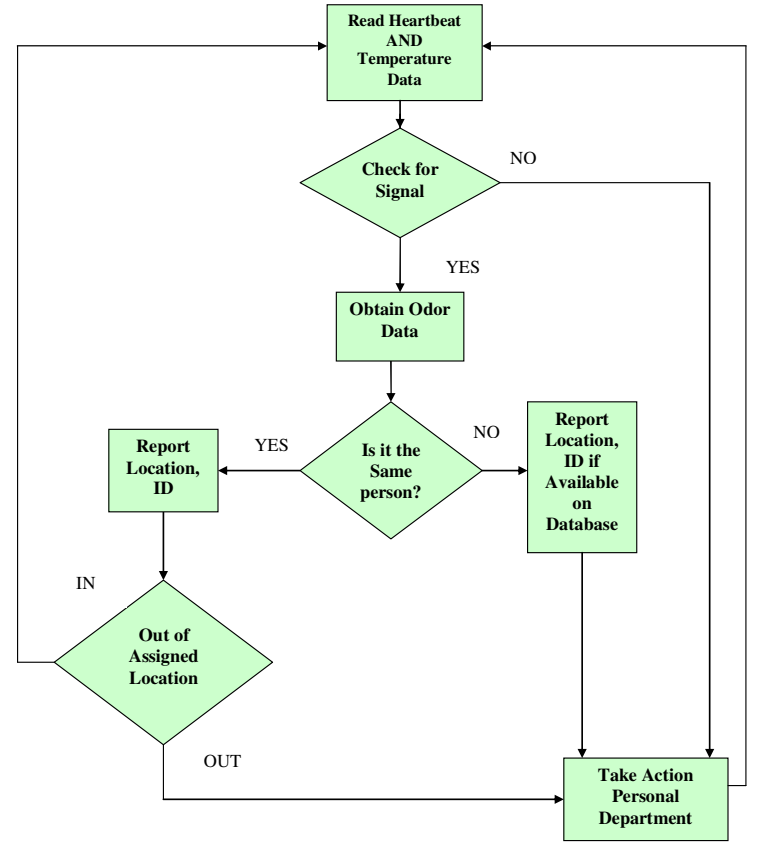

Fig. 4: Processing algorithm

today's complex competitive environment. Of all the devices developed for on-site analysis, the electronic nose is the most versatile in terms of its ease of use and number of potential applications. An Electronic Nose is a vapor analyzer, which provides a recognizable image of specific vapor mixtures (fragrances) containing possibly hundreds of different chemical species. The electronic nose described is a universal detector with very fast response (10 seconds), a large dynamic operating range of vapor concentrations and $\mathrm{ppb}$ sensitivity.

Such odor detecting devices possess the following characteristics:

* No individual sensor elements

* Inexpensive, small, excellent mechanical stability, low power

* Physical differentiation >> collective ageing

* Gradient technique: Reliability checks \& repairs,

Noise reduction without extended measuring time

* Power consumption < 1 Watt

* High chemical \& thermal stability

* Detection limits < 1ppm

Such a small device can be integrated within the transmission pocket or handheld device

2. Temperature Sensor Chip:

3. Heartbeat Sensor Chip

System operation: Figure 4 illustrates the overall operation of the proposed tracking system. The system uses the following operating rules:

* Use signal strength to calculate location using the specified mathematical formulae.
* Use logical AND between Heartbeat and Temperature data to ensure that a person is wearing the device.

* Use odor signature to identify the holder.

* Store the obtained data in a database as a record.

* The data is arranged and stored as [ID, Location, Time].

* The RPT Frame is represented as follows:

\begin{tabular}{|r|r|r|l|}
\multicolumn{1}{|c}{ 1-bit } & \multicolumn{1}{c}{ 8-bits } & 6-bits \\
\hline $\begin{array}{r}\text { Heartbeat } \\
\text { Header }\end{array}$ & $\begin{array}{r}\text { Temp. } \\
\text { Header }\end{array}$ & $\begin{array}{r}\text { Odor } \\
\text { Header }\end{array}$ & Optional \\
& & & \\
\hline
\end{tabular}

\section{DISCUSSION AND CONCLUSION}

The system described here only provides a small framework in which a location sensing can be designed. Further improvements on it can be made quite easily. For instance improving the signal strength measuring, such that a more accurate approach is developed. Finding the area on the map where most of the lines intersect would yield an approximate location of the tagged object. At this point using the proposed system is sufficient as a start. However, more intersections would improve the accuracy of the prediction but would introduce more complexity tool in the systems and require a larger processing overhead. Other improvements to the system could include:

1. Using sensors at arbitrary positions and have them calibrate themselves with respect to a set of reference transmitters at known positions

2. Only sensors that are close enough to the target need to participate and this has to be determined dynamically by group formation.

3. Extend dimensional coverage to three dimensions In this paper we presented a proposed build up of location sensing system using TSE system. Although this system is admittedly not altogether tested, it is a good reflection of how an advanced tracking system works $^{[18-22]}$.

\section{REFERENCES}

1. Gardner, J.W., V.K Varadan and O.O. Awadelkarim, 2001. Microsensors, MEMS and Smart Devices. New York: Wiley.

2. Kumar, S. and D. Shepherd, 2001. SensIT: Sensor information technology for the warfighter. Proc. 4th Intl. Conf. Information Fusion, pp: TuC1-3TuC1-9.

3. Corella, J., 2003. Tactical automated security system (TASS): Air force expeditionary security. Presented at the SPIE Conf. Unattended Ground Sensor Technologies and Applications, Orlando, FL, 2003. 
4. Steere, D., A. Baptista, D. McNamee, C. Pu and J. Walpole, 2000. Research challenges in environmental observation and forecasting systems," in Proc. 6th Int. Conf. Mobile Computing and Networking (MOBICOMM), pp: 292-299.

5. Lee, K., 2001. Wireless sensing and IEEE 1451. Presented at the Sensor Conf./Expo 2001, Chicago.

6. Deb, B., S. Bhatnagar and B. Nath, 2001. A topology discovery algorithm for sensor networks with applications to network management. Dept. Comput. Sci., Rutgers Univ., Tech. Rep. DCS-TR441.

7. Hightower, J. and G. Borriello, 2001. Location systems for ubiquitous computing. IEEE Computer, 34: 57-66.

8. Gupta, P. and P.R. Kumar, 2000. The capacity of wireless networks. IEEE Trans. Inform. Theory, 46: 388-404.

9. Yao, Y. and J.E. Gehrke, 2003. Query processing in sensors networks. Proc. 1st Biennial Conf. Innovative Data Systems Research, Asilomar, CA.

10. Intanagonwiwat, C., R. Govindan, D. Estrin, J. Heidemann and F. Silva, 2002. Directed diffusion for wireless sensor networking. IEEE/ACM Trans. Networking, 11: 2-16.

11. Chong, C.Y., F. Zhao, S. Mori and S. Kumar, 2003. Distributed tracking in wireless ad hoc sensor networks. Proc. 6th Intl. Conf. Information Fusion, pp: 431-438.

12. Zhao, F., J. Shin and J. Reich, 2002. Informationdriven dynamic sensor collaboration for tracking applications. IEEE Signal Processing Mag., 19: 61-72.

13. Shin, J., L.J. Guibas and F. Zhao, 2003. A distributed algorithm for managing multi-target identities in wireless ad-hoc sensor networks. Presented at the 2nd Intl. Workshop Information Processing in Sensor Networks (IPSN'03), Palo Alto.
14. Qi, H., S.S. Iyengar and K. Chakrabarty, 2001. Multi-resolution data integration using mobile agents in distributed sensor networks. IEEE Trans. Syst., Man, Cybern. C, 31: 383-391.

15. Hong, X., K. Xu and M. Gerla, 2002. Scalable routing protocols for mobile ad hoc networks. IEEE Network Mag., 16: 4.

16. Olafsdottir, G., E. Chanié, F. Westad, R. Jonsdottir, C.R. Thalmann, S. Bazzo, S. Labrèche, P. Marcq, F. Lundby and J. Erik Haugen, 2005. Prediction of microbial and sensory quality of cold smoked atlantic salmon (Salmo salar) by electronic nose. J. Food Sci., 70: S563-574.

17. Mészáros, P. and I. Bkae, 2004. Assessment of coffee taste by means of the a ASTREE electronic tongue. Intl. Conf. Food Physics and Dairy Sci., Pecs, Hungary, pp: 67-69.

18. Karp, B. and H.T. Kung, 2000. GPSR: Greedy perimeter stateless routing for wireless networks. Proc. MOBICOM '00, New York.

19. Li, J.J., D.S.J. De Couto, D. Karger and R. Morris, 2000. A scalable location service for geographic ad-hoc routing. Proc. MOBICOM '00, New York.

20. Nagpal, R., H. Shrobe and J. Bachrach, 2003. Organizing a global coordinate system from local information on an ad hoc sensor network. 2nd Intl. Workshop on Information Processing in Sensor Networks (IPSN '03), Palo Alto.

21. Nicolescu, D. and B. Nath, 2001. Ad-hoc positioning systems (APS). Proc. IEEE GLOBECOM '01.

22. Niculescu, D. and B. Nath, 2003. DV based positioning in ad hoc networks. J. Telecommunication Systems 